\title{
Acquisition of the tide-induced Lagrangian residual current field by the PIV technique in the laboratory
}

\author{
Tao Wang • Wensheng Jiang • Xu Chen • Shizuo Feng
}

Received: 2 March 2013 / Accepted: 12 September 2013 /Published online: 25 September 2013

(C) The Author(s) 2013. This article is published with open access at Springerlink.com

\begin{abstract}
In this paper, a new particle image velocimetry (PIV)-based measurement method is proposed to obtain the high-resolution tide-induced Lagrangian residual current field in the laboratory. A long gravity wave was generated to simulate the tide in a narrow tank full of water laden with PIV particles. Consecutive charge-coupled device (CCD) images were recorded with the studied layer illuminated with a laser beam. Two images separated by one tidal period were processed by applying the pattern-matching algorithm to get the horizontal tide-induced Lagrangian residual current field. The results coincide with sporadic results from the traditional surface-float tracing method, but with much higher spatial resolution and accuracy. Furthermore, it is found that the direct acquisition of the Lagrangian residual current may reduce the error at least by one order compared with those acquisition methods that require the detailed information of the tidal cycle.
\end{abstract}

Keywords Lagrangian residual current .

Particle image velocimetry $\cdot$ Eulerian residual velocity ·

Stokes' drift velocity · Mass transport velocity

\section{Introduction}

In bays and estuaries, the tide-induced residual current, which is generated by the nonlinearity of the tidal system, is an important component in coastal and estuarine circulations (Nihoul and Ronday 1975; Feng 1990). Although the discussion of the tide-induced residual current is inevitable when studying the

Responsible Editor: Birgit Andrea Klein

T. Wang $\cdot$ W. Jiang $(\bowtie) \cdot X$. Chen $\cdot$ S. Feng

Physical Oceanography Laboratory, Ocean University of China,

Songling Road 238, Qingdao, Shandong 266100, People's Republic of China

e-mail: wsjang@ouc.edu.cn dynamics of the tide dominant shallow sea systems, its definition is far from unified. As a result, different methods have been proposed to derive it from the tidal current fields. The most frequently used definition is the Eulerian mean velocity, the socalled Eulerian residual velocity, which is defined as the time average or the steady part in the harmonic analysis of the tidal current at one fixed location for one or several tidal periods. The Eulerian mean method was used in many analytical or numerical studies to get the inter-tidal motion (e.g., Lopes and Dias 2007; Huijts et al. 2009; Vaz et al. 2009). However, it is not an appropriate representative of the inter-tidal current because of its violation of the mass conservation (Feng et al. 1984) and the ambiguity of the fictitious dispersion term (Fischer et al. 1979).

Another definition of the tidally averaged velocity is the quotient of the net displacement of a water parcel after several periods and the elapsed time, namely, the Lagrangian residual velocity. Longuet-Higgins (1969) was the first to put forward the concept of the Lagrangian mean velocity in large-scale circulations. He pointed out that the mass transport velocity equaled the sum of the Eulerian residual velocity and the Stokes' drift velocity. Zimmerman (1979) examined the approximation of the rigorous Euler-Lagrangian transformation and defined the Lagrangian residual velocity as the net displacement of a labeled water parcel over one or several tidal periods. Cheng and Casulli (1982) proposed that the Lagrangian residual velocity was a function of the tidal phase when the water parcel was labeled. Feng et al. (1986a) systematically analyzed the depth-averaged dynamic system in the weakly nonlinear shallow sea. They divided the Lagrangian residual velocity into different orders under the assumption of the weakly nonlinear convective terms and found that the first-order Lagrangian residual velocity was the mass transport velocity and the second order of the Lagrangian residual velocity was the Lagrangian residual drift velocity, which displayed the Lagrangian nature of the Lagrangian residual velocity. Furthermore, they established a new inter-tidal mass transport equation, in which the 
convective velocity was the mass transport velocity, making the "tidal dispersion" term in the traditional inter-tidal mass transport equation vanish (Feng et al. 1986b; Cheng et al. 1989). Feng (1987) set the field equations for the Lagrangian residual circulation induced by an $\mathrm{M}_{2}$ tidal system and developed a three-dimensional Lagrangian residual current model.

Dortch et al. (1992) used the model developed by Feng (1987) to simulate the annual salinity distribution in 1985 in the Chesapeake Bay, whose results coincided with the field observation results. This model solved the problem that with the use of the Eulerian residual velocity, the simulated salinity was lower than the observational salinity. Also, based on this model, several studies (Cerco and Cole 1993; Cerco 1995a, b) simulated the multiyear nutrients distribution and the eutrophication distribution trend and found that the results were reasonable. Delhez (1996), after discussing the different approaches used to simulate the long-term advection of passive constituents on tidal shelves in the framework of large-scale hydrodynamic modeling, found that the Eulerian residual transport velocities failed to represent the long-term motions when the tidal nonlinearities were important and concluded that the first-order Lagrangian residual velocity was a very good solution. Wei et al. (2004) used the Hamburg Shelf Ocean Model (HAMSOM) to simulate the Eulerian and Lagrangian residual current in the Bohai Sea. Jiang and Feng (2011) used the analytical method to study the tide-induced Lagrangian residual current in a narrow bay and compared the difference among the Eulerian residual velocity, the Eulerian residual transport velocity, and the Lagrangian residual velocity, demonstrating that the Lagrangian residual current was the appropriate representative of the water mass inter-tidal transport in the tide-dominant area. The above results clearly show that it is appropriate to use the first-order Lagrangian residual velocity to describe the circulation fields in the shallow sea.

All the above results are based on the weakly nonlinear assumptions. Feng et al. (2008) broke this limit and studied the tide-induced circulation and mass transport in the general nonlinear system. They proposed a new concept of the concentration for inter-tidal transport processes, named as the "Lagrangian inter-tidal concentration."

The studies mentioned above prove that the Lagrangian residual current is the appropriate representative of the intertidal current, but it has not been widely accepted up to now. One of the possible reasons is that the mathematics of the Lagrangian residual current is more complex than that used in the Eulerian residual velocity and the accurate acquisition of the difference between them is difficult due to the small value of the residual currents compared with the tidal current.

Generally, there are two ways to get the Lagrangian residual current. One way is to get the sum of the Eulerian residual velocity and the Stokes' drift velocity, and the other is to analyze the trajectories of water parcels. However, it is not an easy task to obtain the Lagrangian residual current field by either method. As mentioned above, the tide-induced residual current is very small and is often of the same order with the instrument measurement error. The direct measurement error of the time series of tidal currents will contaminate the Lagrangian residual current. The trajectory method, which arises from the original definition of the Lagrangian residual current, is reliable, but it is not possible to get enough trajectories to make a satisfying spatial coverage.

Things may be easier in the laboratory because of long history of the experimental fluid dynamics studies. By now, there have been a number of laboratory experimental studies on the tide-induced residual current, but most of them are confined to the obtaining of the Eulerian residual velocities. Yanagi $(1976,1978)$ did a series of laboratory experiments on the Eulerian residual velocity in a square bay model. Yanagi and Yoshikawa (1983) investigated the effects of the Coriolis force and bottom slope on the tide-induced residual current, but they also only acquired the Eulerian residual velocity. Yasuda (1984) obtained the surface Eulerian residual velocity and Lagrangian residual current in a bay with a sloping bed. In all of these studies, the method used to acquire the tide-induced residual current is the surface-float tracing method, i.e., some floats are spread on the surface of the water and the trajectories of the floats are acquired by taking a series of photos in several tidal periods. However, in order to distinguish the floats in the frames acquired by the camera, the distances among these floats must be large enough. Therefore, the velocity can only be obtained at sporadic points and the velocity field can hardly be obtained.

In the 1980s, a method to obtain the high-resolution velocity field, the particle image velocimetry (PIV) technique, was developed in experimental fluid dynamics (Adrian 2005). This technique makes the acquisition of accurate and highresolution instantaneous velocity fields possible. Recently, it has been used in many laboratory studies on ocean phenomena (e.g., Dossmann et al. 2011; Mercier et al. 2011; Wang et al. 2012). In the present study, based on the tide-induced Lagrangian residual current theory, a method that enables the acquisition of the high-resolution Lagrangian residual current field through the PIV technique is proposed. The paper is organized as follows: Section 2 describes the measurement principles, the experimental setup, and the similarity analysis. The results and evaluation are discussed in Section 3. Section 4 analyzes the impact of measurement error on the measurement of the residual current. Conclusions are presented in Section 5.

\section{Measurement principles, experimental setup, and similarity analysis}

\subsection{Measurement principles}

With the classical PIV technique, the particles with a diameter of tens of microns are suspended in the fluid. Illuminating one 
slice of the fluid with a laser beam and taking photos of the slice with a high-speed charge-coupled device (CCD) camera, a sequence of images that reflects the distribution of the particles can be obtained. Then, every frame is split into an array of interrogation windows and a displacement vector for each window can be obtained by processing two consecutive frames with autocorrelation or cross-correlation techniques, which is called the pattern-matching algorithm. Every interrogation window can be regarded as a fluid parcel, and the size of the interrogation window should be chosen to have at least 6 pixels per window on average. As a result, the instantaneous velocity of each fluid parcel is obtained to formulate the highresolution velocity field.

To accurately measure the fluid motion, three conditions should be satisfied. Firstly, the PIV particles should follow the flow of the fluid very well. Secondly, the distances that fluid parcels move during the time interval between two neighboring frames should not be too long to hinder identifying the movement of the fluid parcel defined by the interrogation window. Thirdly, the distribution of the particles in a fluid parcel, i.e., an interrogation window, should remain almost unchanged between the two frames used for calculating. The third problem is called the particle pattern decorrelation which is of major concern in acquiring the velocity field by applying the PIV technique. Two mechanisms contribute to this phenomenon: one is the effect of the diffusion, which can change the particle distribution pattern in one water parcel; the other is the velocity normal to the plane being studied.

According to the definition, the Lagrangian residual velocity can be obtained by processing the two images separated by one tidal period with pattern-matching algorithm if the above three conditions are satisfied. In the present study, the PIV particles are chosen as those used in similar studies; then, the first condition is satisfied easily. According to the behavior of the tide-dominant movement, the fluid parcels will come back to the positions near their initial positions after one or several tidal periods. Therefore, the net displacements of water parcels in one or several tide periods are small and may be measured by the PIV technique, which will be discussed in the following part. As for the particle pattern decorrelation, velocity normal to the illuminated plane can be estimated to evaluate its influence, but the effect of the turbulent diffusion can only be discussed after the experiment is carried out.

\subsection{Experimental setup}

This experiment setup is based on a narrow bay model with an analytical solution. Winant (2008) first gave the analytical solution of the first-order Lagrangian residual velocity for a parabolic depth profile by calculating the Eulerian residual velocity and the Stokes' drift velocity separately. The present author established the governing equations for the first-order Lagrangian residual velocity and solved it analytically (Jiang and Feng under review). In the above models, the momentum equation of the vertical direction is reduced to hydrostatic equilibrium because of the small ratio of depth to horizontal spatial scale when studying the tidal currents in a bay. This ratio is of the order of $10^{-3}$ at most which is difficult to reproduce in the laboratory without changing its dynamics.

In the present study, the laboratory experiments were conducted in a rectangular tank of 5,000 $\mathrm{mm}$ long ( $x$-axis), $150 \mathrm{~mm}$ wide ( $y$-axis), and $400 \mathrm{~mm}$ high ( $z$-axis). At the lefthand side of the tank, a wave generator was used to generate gravity waves with a period of $7.6 \mathrm{~s}$ to simulate tides. At the right-hand side, a bottom slope was put in the tank which was $1,000 \mathrm{~mm}$ in length and made the water depth decreasing linearly along the $y$-coordinate from 120 to $40 \mathrm{~mm}$ (Fig. 1), i.e., the mean depth of the water on the slope was $80 \mathrm{~mm}$. The measurement area was chosen at the right-hand side of the tank which was $420 \mathrm{~mm}$ long. The laser beam illuminated a horizontal plane just below the water surface. The PIV particles with a diameter of about $30 \mu \mathrm{m}$ were suspended in the water. At about $1 \mathrm{~m}$ above the water surface, a camera with a frame rate of 30 fps was set to record the particle distributions of the illuminated plane with a resolution of $1,392 \times 1$, 040 pixels.

\subsection{Similarity analysis}

First, the properties of the wave in this experiment are diagnosed. In this experiment, the elevation of the water $\zeta$ is of the order of $1 \mathrm{~mm}$, which is small compared with the water depth, so the dispersion relations can be written as $\omega^{2}=g k \operatorname{th}(h k)$, where $\omega, k$, and $h$ represent the angular frequency, the wave number, and the mean water depth, respectively. According to the equation $k=\frac{2 \pi}{\lambda}$, where $\lambda$ denotes the wavelength, it can be deduced that the ratio of the water depth to the wavelength is of the order of 0.01 . Therefore, the wave generated in this experiment is a long gravity wave, whose vertical velocity is

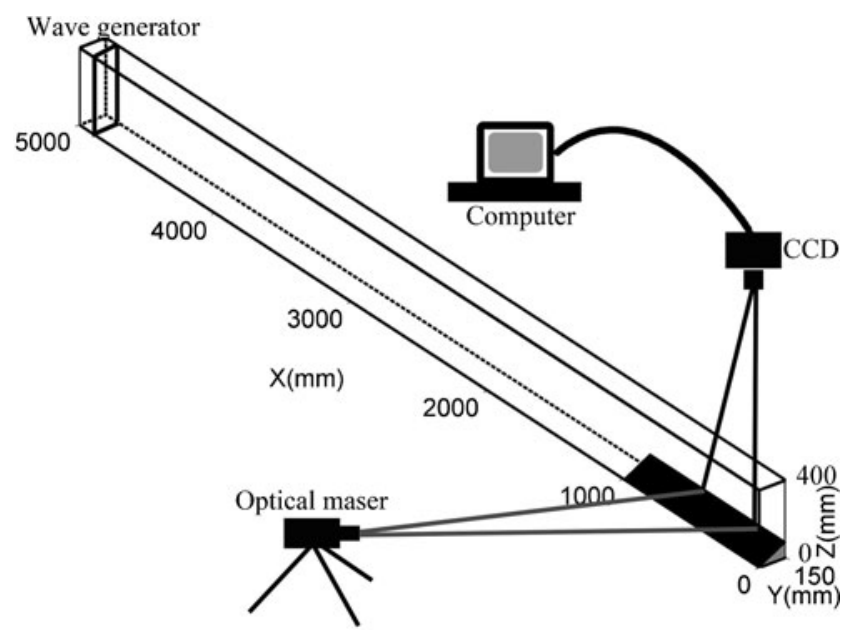

Fig. 1 The experimental setup 
much smaller than longitudinal velocity. This ensures that the vertical velocity will not affect the distribution of the PIV particles in any horizontal plane, which partly satisfies the third condition mentioned in Section 2.1.

Second, based on the experimental setup, the independent characteristic scales are presented, namely, the basin length scale $L \sim 10 \mathrm{~m}$, the basin width scale $B \sim 0.1 \mathrm{~m}$, the basin depth scale $H \sim 0.1 \mathrm{~m}$, and the time scale $T \sim 10 \mathrm{~s}$. According to the measurement results, the peak longitudinal current is about $10 \mathrm{~mm} / \mathrm{s}$, i.e., the longitudinal velocity scale $U \sim 10 \mathrm{~mm} / \mathrm{s}$. Because $\frac{B}{L}=0.01$ and $\frac{H}{L}=0.01$, the experimental area is geometrically similar to a narrow shallow bay.

According to the above analysis, the second condition mentioned in Section 2.1 is satisfied in this experiment: because $\frac{U T}{L}<<1$, the experiment setup defines a weakly nonlinear system, where UT means that the characteristic value of water parcel excursion is about $0.1 \mathrm{~m}$. In addition, in the weakly nonlinear system, the ratio of the residual current to the tidal current is also equal to $\frac{U T}{L}$ (Ianniello 1977; Feng et al. 1986a). Therefore, the net displacement of the water parcel over one period is of the order of $0.001 \mathrm{~m}$. The fluid parcels will be back to the positions near their initial positions after one tidal period. According to the measurement area and the CCD resolution, the net displacement of the order of $0.001 \mathrm{~m}$ can be measured through the PIV technique.

As to the third condition, it is assumed that the horizontal convection dominates in the experiment, i.e., the PIV particles' distribution in a horizontal interrogation window holds well in one tidal period. If this assumption is right, the highresolution horizontal Lagrangian residual current field on a certain layer can be obtained rigorously by applying the pattern-matching algorithm on the initial and final frames over one tidal period. Yet the direct estimation of the importance of the turbulence diffusion in an interrogation window is beyond the scope of the present study because it is of the subgrid scale here. So in the following section, this method will be evaluated by analyzing the experiment results.

\section{Results and evaluation}

\subsection{Results}

In this experiment, 228 frames can be acquired in one tidal period. The phase when the ebb tidal velocity reaches its maximum is assumed to be $\varphi=0$. In the experiment, the images of three periods are taken. Then, if the patternmatching algorithm is applied to each pair of frames separated by one tidal period, the high-resolution Lagrangian residual current fields with different initial phases are obtained. Replicating experiments have also been done, and Lagrangian residual current fields are nearly the same. Averaging these results with the same initial phase, the Lagrangian residual current fields with the different phases are displayed in Fig. 2.

It can be seen in Fig. 2 that when the tidal current flows over the narrow bay with the bottom slope decreasing linearly along the width, the Lagrangian residual current flows in at the shallow side and flows out at the deep side. The residual current is around $0.1 \mathrm{~mm} / \mathrm{s}$, and the tidal velocity amplitude is about $10 \mathrm{~mm} / \mathrm{s}$. Therefore, the residual current is one to two orders in magnitude smaller than the tidal velocity. The pattern of the Lagrangian residual current field does not change with the initial phase. This is because the experiment setup defines a weakly nonlinear system; the second-order Lagrangian residual current which is dependent on the phase of tidal current is much smaller than the first-order Lagrangian residual current (Feng et al. 1986a). The Lagrangian residual current field for a tidal period of $5 \mathrm{~s}$ is also obtained and is similar to that in Fig. 2 (figures are omitted for brevity).

This is in accordance with the result of the analytical model when the viscosity coefficient is taken to be very small to make the turbulent viscosity force two orders smaller than the inertial force (see Fig. 3). It should be noted that the equations of the analytical model are derived based on the shallow-water approximation because the water depth is at least three orders smaller than the horizontal spatial scale in a real bay. But limited by the experimental technique, the water depth is of the same order as the bay width in the present experiment though it is one to two orders smaller than the bay length. Furthermore, the analytical result in Fig. 3 is only the firstorder approximation to the Lagrangian residual current which is independent of the initial phase. Thus, the experimental results can only be compared with the analytical results qualitatively.

The Lagrangian residual velocity can also be obtained by processing the two images separated by two or more tidal periods. The results show that the Lagrangian residual currents cannot be determined at some interrogation windows which means that the pattern decorrelation becomes important if the time interval between the frame of images is too long.

\subsection{Error analysis}

Although the spatial resolution of the camera is $1,392 \times 1,040$, the spatial resolution of the measurement area in the tank is 1 , $392 \times 485$ because the measurement area covers only part of the frame. Considering the geometry scale of the measurement area, it can be found that a pixel is equal to about $0.31 \mathrm{~mm}$ in the present experiment setup. The software for calculation used in this paper is DigiFlow (Dalziel Research Partners 2008). In this experiment, the accuracy is set to best when processing the data by using DigiFlow, in which the calculated accuracy of the net displacement through PIV is about $1 / 100$ of a pixel which is $3.1 \times 10^{-3} \mathrm{~mm}$. Because the tidal period in the experiment is $7.6 \mathrm{~s}$, and there are 228 frames in one period, 
Fig. 2 The Lagrangian residual current fields obtained by the PIV technique. The initial phase is a 0 , b $\pi / 2$, c $\pi$, and $\mathbf{d} 3 \pi / 2$

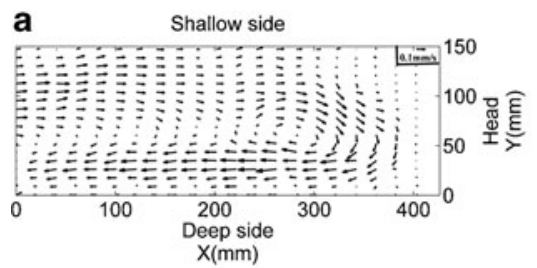

$\varphi=0$

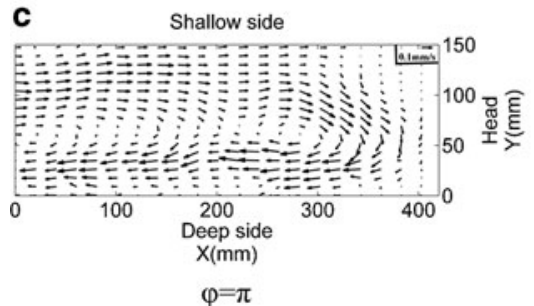

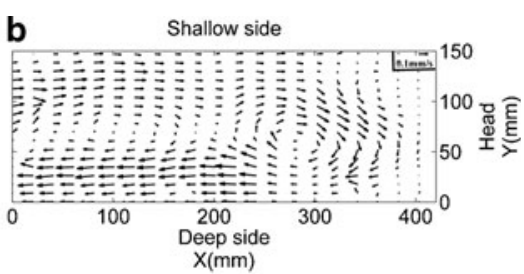

$\varphi=\pi / 2$

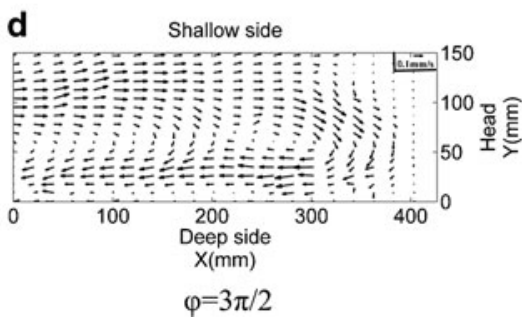

the error is about $9.3 \times 10^{-2} \mathrm{~mm} / \mathrm{s}$ of the instantaneous velocities and is about $4.1 \times 10^{-4} \mathrm{~mm} / \mathrm{s}$ of the Lagrangian residual velocities. Therefore, the measurement error of the PIV method in the present experiment is two orders smaller than the measured variables.

\subsection{Comparison with the surface-float tracing method}

According to its definition, the Lagrangian residual current can describe the net displacement of the water parcel after one or several tidal periods. To evaluate the Lagrangian residual current field obtained by the PIV technique, the traditional surface-float tracing method was used as a reference. The surface-float tracing method has been used by some researchers (Yanagi 1976, 1978; Yanagi and Yoshikawa 1983; Yasuda 1984), and it was employed again in this study to obtain the Lagrangian residual current at some specific points with the same experimental conditions as in the PIV experiment. When the PIV technique was applied to get the current field, 30 surface floats with a diameter of about 2-3 $\mathrm{mm}$ were spread on the water surface simultaneously. Just considering the initial and final positions of these surface floats over one period, the net displacements of the floats over one period can be obtained. Taking $\varphi=0$ as the initial time and dividing these net displacements by one tidal period, the Lagrangian residual current at these initial points is presented in Fig. 4.

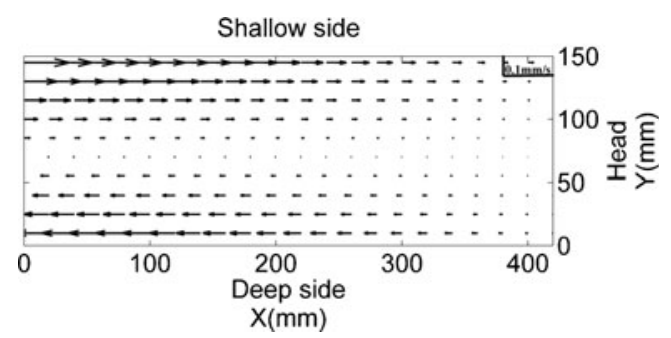

Fig. 3 The first-order Lagrangian residual current field obtained by an analytical method
The Lagrangian residual current in Fig. 4 exhibits the same flow pattern as that in Fig. 2. This coincides with that of Yasuda (1984), who also used the surface-float tracing method to obtain the Lagrangian residual current in a narrow bay model with a sloping bed along the transverse direction.

However, the velocities in Fig. 4 are not exactly equal to those in Fig. 2. According to the surface-float tracing method, the positions of these floats are determined by identifying the high-brightness area in the two frames as shown in Fig. 5a. The contiguous pixels with a brightness value greater than 0.5 are regarded as being occupied by the floats. The geometrical center of the float can be obtained by averaging the pixel positions occupied by each float. Assuming the float to be a sphere, as shown in Fig. 5a, the geometrical center of the float can be regarded as the center of a circle, and the largest distance between the geometrical center and the edge can be regarded as the radius. Actually, the floats are not exactly spherical and one float only occupies several pixels, which means that the shape of the same float may not be the same in different frames. So there exist positioning errors for center positions of these floats in different frames, which is of the same order as the difference between the radii of the floats in the initial frame and final frame.

In Fig. 5b, one float starting from the pixel $(575,114)$ is taken as an example to analyze the net displacement over one

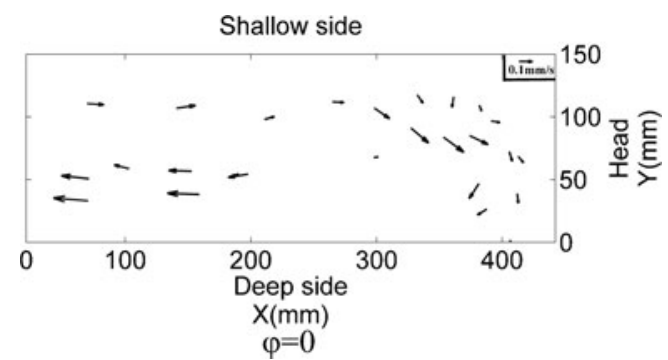

Fig. 4 The Lagrangian residual current obtained by the surface-float tracing method. The initial phase is 0 

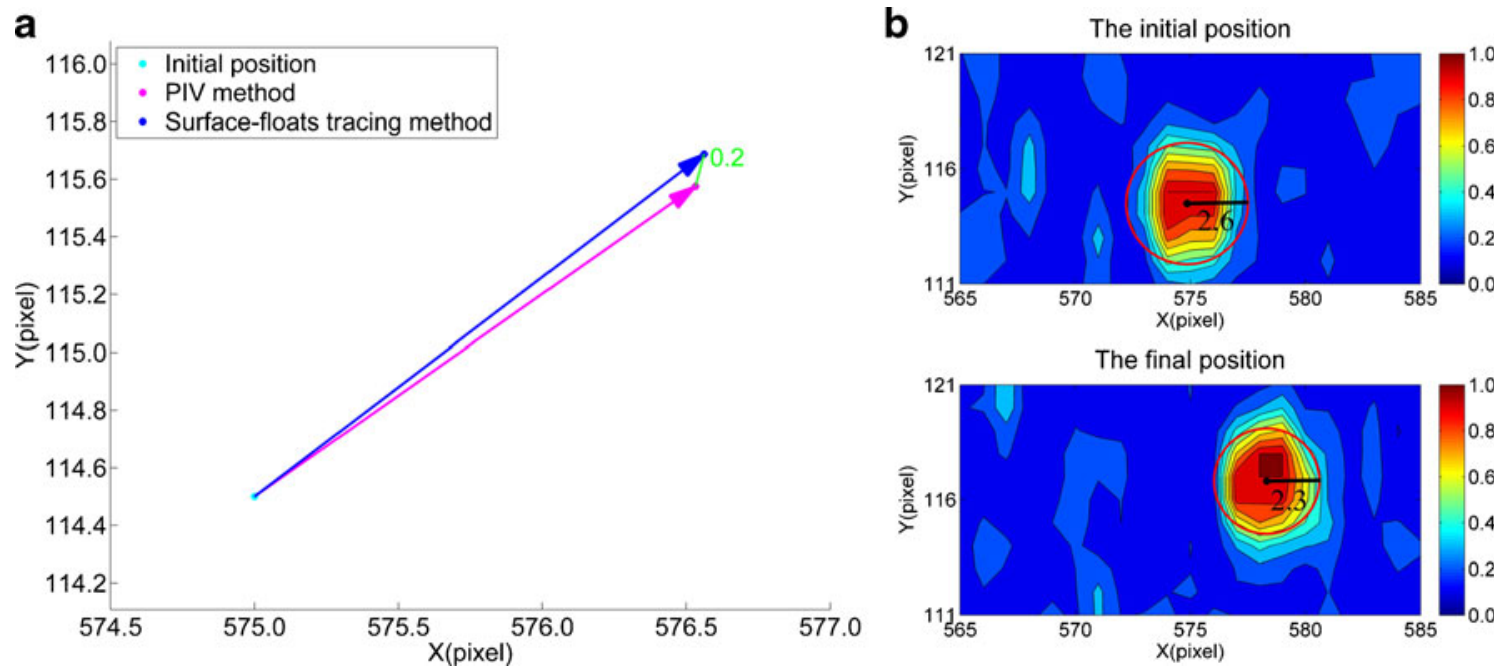

Fig. 5 a Net displacements over one tidal period. b Positioning error for the center position of the float in the surface-float tracing method

tidal period obtained by both the surface-float tracing method and the PIV method. Firstly, the angle difference between the directions of both displacements is approximately $1^{\circ}$, which is small enough to demonstrate that there exists little difference between the directions of the Lagrangian residual currents obtained by these two methods. Secondly, the difference in magnitude between the net displacements is nearly 0.2 pixels. Figure 5a shows that the positioning error of the float is
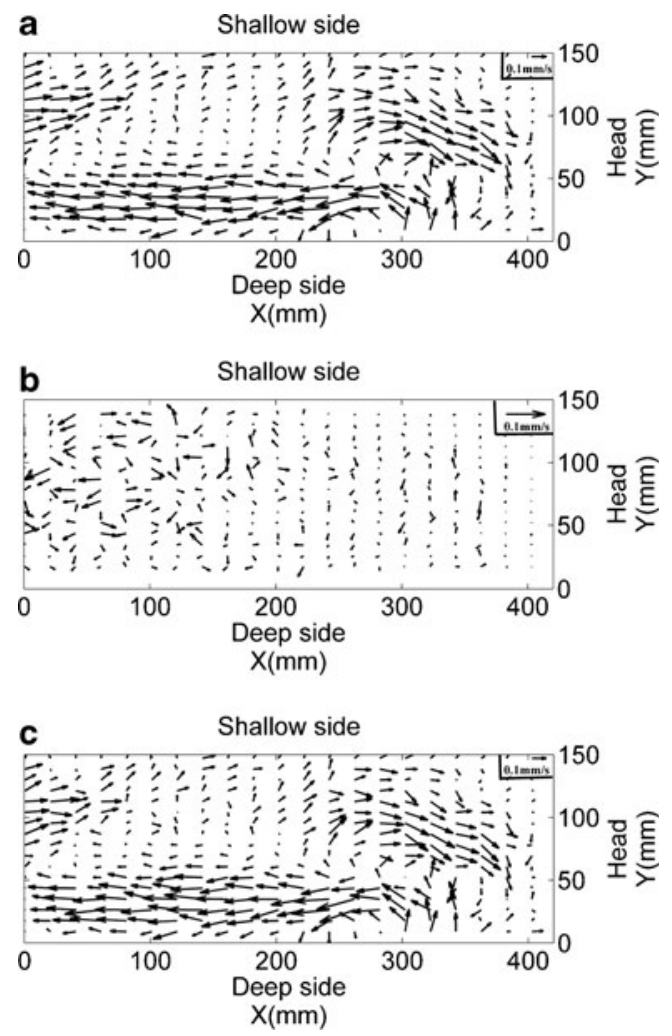

Fig. 6 a The Eulerian residual velocity, b the Stokes' drift velocity field, and $\mathbf{c}$ the Lagrangian residual current filed obtained by calculating the sum of the Eulerian residual velocity and the Stokes' drift velocity
0.3 pixels, which is of the same order as the difference between these two methods. Therefore, the difference in magnitude between the net displacements can be attributed to the positioning error of the surface float.

The angle and displacement differences between these two methods and the positioning errors in surface-float tracing method were calculated for all the 30 floats. The averaged angle and displacement difference between these two methods are $6^{\circ}$ and 0.4 pixels respectively, and the averaged positioning error is 0.3 pixels. This shows that the angle is very small and the mean displacement difference is of the same order as the mean positioning error. Therefore, the difference between these two methods is mainly caused by the positioning errors of the center positions of the floats. In addition, the floats are too large to follow the flow well, which may also explain why the surface-float tracing method resulted in a larger measurement error than the PIV method.

Therefore, it is feasible to use the PIV method to acquire the high-resolution Lagrangian residual current field in the laboratory. Moreover, the PIV method ensures greater accuracy than the surface-float tracing method. Thirdly, with the PIV method, the particles suspend in the fluid, which makes the acquisition of the three-dimensional structure of the Lagrangian residual current possible.

\section{The impact of measurement error on the residual current}

It has been proved that under weakly nonlinear conditions, the first-order Lagrangian residual velocity equals the sum of the Eulerian residual velocity and the Stokes' drift velocity (Longuet-Higgins 1969; Feng et al. 1986a). This proposes another approach to obtain the first-order Lagrangian residual current, which is often used in the numerical studies (e.g., 
Dortch et al. 1992; Delhez 1996; Jiang and Sun 2002; Hainbucher et al. 2004; Wei et al. 2004). The Eulerian residual velocity (Fig. 6a) in turn can be obtained by averaging the tidal velocities in one tidal period, and the Stokes' drifts velocity (Fig. 6b) can be obtained according to the formula $\mathbf{U}_{\mathbf{S}}=\overline{\left(\int_{t_{0}}^{t} \mathbf{U} d t \cdot \nabla\right) \mathbf{U}}$, which depends on the time series of the tidal current at one fixed point and the time series of the gradient of the velocity at the same point. The over bar represents the tidal average operator defined as $\overline{\mathbf{f}}=\frac{1}{n T} \int_{t_{0}}^{t_{0}+n T} \mathbf{f}(x, y, t) d t$. The present experiment, however, uses the PIV technique to get the instantaneous tidal velocity fields at a time interval of $1 / 30 \mathrm{~s}$. The first-order Lagrangian residual current acquired by this approach is shown in Fig. 6c.

It can be seen that the pattern of the Lagrangian residual current in Fig. 6c is similar to that in Fig. 2, but it is discontinuous in the left part of the shallow side and the velocity field is more irregular than that in Fig. 2. This is because the measurement errors in the tidal velocities seriously contaminate the residual current in this approach.

In the PIV technique, the instantaneous tidal velocity is obtained by the quotient of the displacement of one interrogation window and the time interval. Obviously, there are measurement errors in determining the displacements. Because the instrument and the experimental setup are fixed, it is assumed that all the displacement vector measurement errors are within the same range and can be represented by $\left(\sigma_{x}, \sigma_{y}\right)$. It is assumed that the number of instantaneous tidal velocity fields in one tidal period is $N$ and the displacement can be denoted as $\left(\Delta x_{k}, \Delta y_{k}\right)$ for the $k$ th time step. Then, the Eulerian residual velocity can be obtained through the equation $\left(u_{E}, v_{E}\right)=\frac{1}{N} \sum_{k=1}^{N} \frac{\left(\Delta x_{k}, \Delta y_{k}\right) \pm\left(\sigma_{x}, \sigma_{y}\right)}{\Delta t}$ with the error being $\left(\sigma_{x}, \sigma_{y}\right) / \Delta t$. Therefore, the minimal error for the Lagrangian residual current obtained through the equation $\left(u_{L}, v_{L}\right)=\left(u_{E}\right.$, $\left.v_{E}\right)+\left(u_{S}, v_{S}\right)$ is at least $\left(\sigma_{x}, \sigma_{y}\right) / \Delta t$.

However, if the Lagrangian residual velocity is calculated through dividing the net displacement in one tidal period by the tidal period and the net displacement of a fluid parcel is assumed as $\left(\Delta x_{L}, \Delta y_{L}\right)$, the formula will be $\left(u_{L}, v_{L}\right)=\frac{\left(\Delta x_{L}, \Delta y_{L}\right) \pm\left(\sigma_{x}, \sigma_{y}\right)}{N \Delta t}$ with the error being $\left(\sigma_{x}, \sigma_{y}\right) /$ $(N \Delta t)$, whose magnitude is $1 / N$ of the error in the former approach. When $N=228$, which is the number of frames in the present experiment, the error arising from the former approach is two orders larger than the error resulting from the approach dividing the net displacement by the tidal period. Moreover, the influence of the measurement error caused by the former approach will be fatal, especially when the residual current is very small.

In in situ observation analysis, this problem may also arise. Generally, the traditional current measurement error is about $0.01 \mathrm{~m} / \mathrm{s}$, so if the Eulerian residual velocity and the Stokes' drift velocity are added together to get the Lagrangian residual current, the minimal error in the Lagrangian residual current will be about $0.01 \mathrm{~m} / \mathrm{s}$. However, in most cases, the order of the Lagrangian residual current is $0.01 \mathrm{~m} / \mathrm{s}$. Therefore, the measurement error will influence the results vitally. On the other hand, if the Lagrangian residual current is obtained by tracking drifters that fully behave like the surrounding water particles, the major error will come from the positioning process. It is quite common to get a positioning accuracy at the order of $10 \mathrm{~m}$, and this will make the Lagrangian residual current error fall at the order of $10^{-4} \mathrm{~m} / \mathrm{s}$ if $\mathrm{M}_{2}$ tide is assumed, causing the error to be at least one order smaller than the Lagrangian residual current itself. Therefore, in the field observation, the drifter tracking method is a good practice to get the Lagrangian residual current.

\section{Conclusions}

In the present experiment, a long gravity wave is generated in a 5-m-long water tank to simulate the tide. According to the tidal oscillatory behavior that the fluid parcels will be back to the vicinity of their initial positions after one tidal period, a method to obtain the high-resolution tide-induced Lagrangian residual current by the PIV technique in the laboratory is proposed. A comparison with the traditional surface-float tracing method demonstrates that this method is feasible and better than the traditional method. Furthermore, it is found that in laboratory experiments and in situ observation analysis, the method of direct acquisition of the Lagrangian residual current may reduce the error by at least one order compared with those acquisition methods that require the detailed information of the tidal cycle. Although it seems difficult to extend this idea to the field research, the error analysis of the present method hints that the surface drifter tracing is better than adding up the Eulerian residual velocity and the Stokes' drift velocity to acquire the first-order Lagrangian residual velocity in the field.

The result in this paper proves that the analytical solution under similar condition as the laboratory experiment is reasonable. In future, the measurement method will be used in laboratory to study the Lagrangian residual current under more realistic conditions.

Acknowledgments This work is supported by the National Natural Science Foundation of China (projects 40976003 and 40906001) and the Scholarship Award for Excellent Doctoral Student granted by the Ministry of Education of China. Tao Wang acknowledges the financial support from the Chinese Scholarship Council.

Open Access This article is distributed under the terms of the Creative Commons Attribution License which permits any use, distribution, and reproduction in any medium, provided the original author(s) and the source are credited. 


\section{References}

Adrian RJ (2005) Twenty years of particle image velocimetry. Exp Fluids 39(2):159-169. doi:10.1007/s00348-005-0991-7

Cerco CF (1995a) Simulation of long-term trends in Chesapeake Bay eutrophication. J Environ Eng 121:298-310

Cerco CF (1995b) Response of Chesapeake Bay to nutrient load reductions. J Environ Eng 121:549-557

Cerco CF, Cole T (1993) Three-dimensional eutrophication model of Chesapeake Bay. J Environ Eng 119:1106-1125

Cheng RT, Casulli V (1982) On Lagrangian residual currents with applications in South San Francisco Bay, California. Water Resour Res 18(6):1652-1662

Cheng RT, Feng S, Xi P (1989) On inter-tidal transport equation. In: Neilson BJ, Kuo A, Brubaker J (eds) Estuarine circulation. Humana, Clifton, pp 133-156

Dalziel Research Partners (2008) DigiFlow user guide. Dalziel Research Partners, Cambridge

Delhez EJM (1996) On the residual advection of passive constituents. J Mar Syst 8:147-169

Dortch MS, Chapman RS, Abt SR (1992) Application of three-dimensional Lagrangian residual transport. J Hydraul Eng 118:831-848

Dossmann Y, Paci A, Auclair F, Floor JW (2011) Simultaneous velocity and density measurements for an energy-based approach to internal waves generated over a ridge. Exp Fluids 51(4):1013-1028. doi:10. 1007/s00348-011-1121-3

Feng S (1987) A three-dimensional weakly nonlinear model of tideinduced Lagrangian residual current and mass-transport, with an application to the Bohai Sea. In: Nihoul JCJ, Jamart BM (eds) Three dimensional models of marine and estuarine dynamics, vol 45, Elsevier oceanography series. Elsevier, Amsterdam, pp 471488

Feng S (1990) On the Lagrangian residual velocity and the mass-transport in a multi-frequency oscillatory system. In: Cheng RT (ed) Coastal and estuarine studies, vol 38. Springer, Berlin, pp 34-48

Feng S, Xi P, Zhang S (1984) The baroclinic residual circulation in shallow seas. Chin J Oceanol Limnol 2:49-60

Feng S, Cheng RT, Xi P (1986a) On tide-induced Lagrangian residual current and residual transport: 1. Lagrangian residual current. Water Resour Res 22:1623-1634

Feng S, Cheng RT, Xi P (1986b) On tide-induced Lagrangian residual current and residual transport: 2. Residual transport with application in South San Francisco Bay. Water Resour Res 22:1635-1646

Feng S, Ju L, Jiang W (2008) A Lagrangian mean theory on coastal sea circulation with inter-tidal transports I. Fundamentals. Acta Ocean Sin 27(6):1-16

Fischer HB, List EJ, Koh R, Imberger J, Brook NH (1979) Mixing in inland and coastal waters. Academic, New York

Hainbucher D, Wei H, Pohlmann T, Sündermann J, Feng S (2004) Variability of the Bohai Sea circulation based on model calculations. J Mar Syst 44(3-4):153-174. doi:10.1016/j. jmarsys.2003.09.008

Huijts KMH, Schuttelaars HM, de Swart HE, Friedrichs CT (2009) Analytical study of the transverse distribution of along-channel and transverse residual flows in tidal estuaries. Cont Shelf Res 29(1):89-100. doi:10.1016/j.csr.2007.09.007

Ianniello JP (1977) Tidally induced residual currents in estuaries of constant breadth and depth. J Mar Res 35(4):755-786

Jiang W, Feng S (2011) Analytical solution for the tidally induced Lagrangian residual current in a narrow bay. Ocean Dyn 61(4): 543-558. doi:10.1007/s10236-011-0381-z

Jiang W, Sun WX (2002) Three dimensional tide-induced circulation model on a triangular mesh. Int J Numer Methods Fluids 38:555566. doi:10.1002//d.231

Longuet-Higgins MS (1969) On the transport of mass by time-varying ocean currents. Deep Sea Res 16:431-447

Lopes JF, Dias JM (2007) Residual circulation and sediment distribution in the Ria de Aveiro lagoon, Portugal. J Mar Syst 68(3-4):507-528. doi:10.1016/j.jmarsys.2007.02.005

Mercier M, Peacock T, Saidi S, Viboud S, Didelle H, Gostiaux L, Sommeria J, Dauxois T, Helfrich K (2011) The Luzon Strait experiment. Paper presented at the 7 th international symposium on strait, Rome

Nihoul I, Ronday F (1975) The influence of the tidal stress on the residual circulation. Tellus A 27:484-489

Vaz N, Miguel Dias J, Chambel Leitão P (2009) Threedimensional modelling of a tidal channel: the Espinheiro Channel (Portugal). Cont Shelf Res 29(1):29-41. doi:10.1016/ j.csr.2007.12.005

Wang T, Chen X, Jiang W (2012) Laboratory experiments on the generation of internal waves on two kinds of continental margin. Geophys Res Lett 39(4), L04602. doi:10.1029/2011gl049993

Wei H, Hainbucher D, Pohlmann T, Feng S, Suendermann J (2004) Tidal-induced Lagrangian and Eulerian mean circulation in the Bohai Sea. J Mar Syst 44(3-4):141-151. doi:10.1016/j. jmarsys.2003.09.007

Winant CD (2008) Three-dimensional residual tidal circulation in an elongated, rotating basin. J Phys Oceanogr 38(6):1278-1295. doi: 10.1175/2007jpo3819.1

Yanagi T (1976) Fundamental study on the tidal residual circulation I. J Oceanogr Soc Jpn 32:199-208

Yanagi T (1978) Fundamental study on the tidal residual circulation II. J Oceanogr Soc Jpn 34:67-72

Yanagi T, Yoshikawa K (1983) Generation mechanisms of tidal residual circulation. J Oceanogr Soc Jpn 39:156-166

Yasuda H (1984) Horizontal circulations caused by the bottom oscillatory boundary layer in a bay with a sloping bed. J Oceanogr Soc Jpn 40: 124-134

Zimmerman JTF (1979) On the Euler-Lagrange transformation and the stokes drift in the presence of oscillatory and residual currents. Deep-Sea Res 26A:505-520 